\title{
Optical studies of GaAs quantum wells strained to GaP
}

\author{
J. A. Prieto, G. Armelles, M.-E. Pistol, ${ }^{a}$ P. Castrillo, ${ }^{\text {b) }}$ J. P. Silveira, and F. Briones \\ Instituto de Microeletrónica de Madrid, Consejo Superior de Investigaciones Científicas, Isaac Newton 8, \\ Parque Tecnológico de Madrid, E-28760 Tres Cantos (Madrid), Spain
}

(Received 26 November 1996; accepted for publication 22 April 1997)

Quantum wells of GaAs lattice matched to GaP have been studied by photoluminescence and electroreflectance. The quantum well thickness was varied between 1 and 6 monolayers in steps of 1 monolayer. Electron-hole transitions have been observed involving states both in the $X$ band and in the $\Gamma$ band, which have been modeled using a k.p. model in conjunction with the envelope function approximation. Overall agreement between theory and experiment is found using an unstrained valence band offset of $0.6 \mathrm{eV}$. (C) 1997 American Institute of Physics.

[S0003-6951(97)04825-0]

In strained semiconductor structures the lattice parameter and the band gap are independent variables. ${ }^{1}$ This is also true for quarternary alloys and in conjunction with strain, many degrees of freedom are available for the engineer. Strained semiconductor structures are essential in important electronic and optoelectronic devices such as strained-layer modulation-doped high-mobility field effect transistors with a very high cut-off frequency ${ }^{2}$ and lasers with very low threshold currents. ${ }^{3,4}$

Most of the work has been performed on semiconductors having a direct band gap. Work on strained materials having indirect band gaps such as $\mathrm{Si}-\mathrm{Ge},{ }^{5}$ AlAs-InAs, ${ }^{6}$ and $\mathrm{GaP}-\mathrm{GaAsP}^{7,8}$ has also been performed. We will report in this letter on the optical properties of GaAs quantum wells (QWs) of strained to GaP, as a natural extension of the work published in Refs. 7 and 8. We have studied electronic transitions related to both the $X$ minima and to the $\Gamma$ minimum of the conduction band, using photoluminescence (PL) and electroreflectance (ER), respectively. The transition energies have been modeled using an eight-band k.p. model, in conjunction with the envelope function approximation. ${ }^{9}$

The samples were grown by atomic layer molecular beam epitaxy $(\mathrm{ALMBE})^{10}$ on undoped $(001) \pm 0.5^{\circ}$ oriented GaP substrates. Each sample contains three GaAs layers of equal thickness, ranging from 1 to 6 monolayer (ML), separated by sufficiently wide ( $38 \mathrm{~nm}$ ) GaP barriers to assure no coupling between adjacent GaAs well layers. The lattice mismatch is $3.6 \%$ and the critical thickness for dislocation formation should be of the order of $6 \mathrm{ML}$, close to the observed value for GaP grown on GaAs. ${ }^{11}$ The growth rate was $1 \mu \mathrm{m} / \mathrm{h}$ and the growth temperature was $400{ }^{\circ} \mathrm{C}$. Observed reflection high-energy electron diffraction patterns correspond to a clear two-dimensional growth mode. In all the samples, an initial GaP buffer layer with a thickness of $0.7 \mu \mathrm{m}$ was grown by molecular beam epitaxy (MBE). The confined phonon frequencies of the samples have been measured by Raman spectroscopy. ${ }^{12}$ The experimental frequencies agree very well with theoretical calculations assuming fully strained GaAs layers and a moderate degree of intermixing at the interfaces. The best agreement is obtained as-

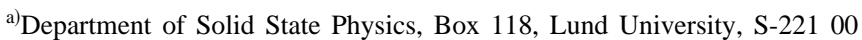
Lund, Sweden. Electronic mail: Mats-Erik.Pistol@ftf.lth.se

${ }^{b}$ Also with Department of Electronics, University of Vallodolid, Dr. Mergelina s/n, E-47011 Valladolid, Spain.
}

suming an As carry-over at the upper interface (probably induced by strain), with a segregation coefficient of about 0.5 .

Figure 1 shows the calculated potential structure of GaAs biaxially strained to GaP. The calculation is based on deformation potential theory, using six bands for the valence band and treating the $X$ band and the $\Gamma$ band separately. ${ }^{13}$ The parameters for the calculation are taken from Ref. 14, with the exception of the hydrostatic deformation potential of the band gap $\left(a_{g}\right)$ of GaAs, which was taken to be $-8.5 \mathrm{eV}$ in order to fit the data in Ref. 15. We assume that the valence band offset does not depend on hydrostatic strain. The few experiments ${ }^{16}$ that have addressed this question agree with this assumption, but more work is needed for general conclusions to be drawn about this important parameter. An unstrained valence band offset of $0.6 \mathrm{eV}$ (giving a strained valence band offset of $0.7 \mathrm{eV}$ ) was used in the figure, which gives the best fit to the experiment, as will be shown later. From now on we will use the abbreviation VBO for the unstrained valence band offset. It can be seen that the GaAs layer is indirect in $\mathbf{k}$ space, due to the strain. The lowest conduction band minima are the $X_{x y}$ minima in which the $\mathbf{k}$ vector is in the layer plane. The $X_{z}$ conduction band minimum with the $\mathbf{k}$ vector is in the growth direction and appears at higher energies. For a VBO of $0.6 \mathrm{eV}$ the discon-

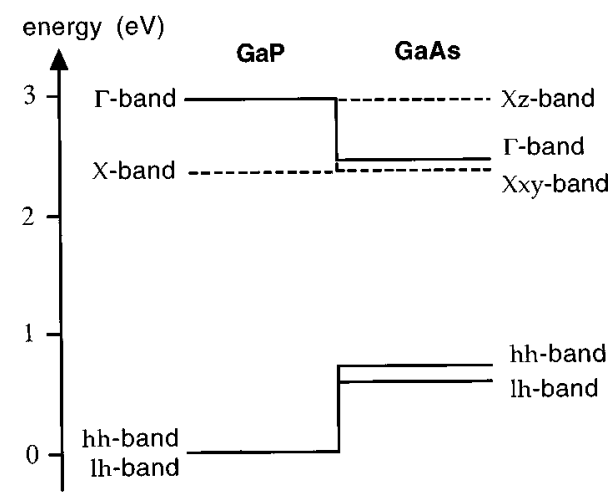

FIG. 1. A diagram of the band-edges at $k=0$ for the heavy hole and the light hole in the valence band, as well as the $\Gamma$ minimum and the $X$ minima of the conduction band for GaAs strained to GaP. The $X$ minima in GaAs are split into $X_{x y}$ and $X_{z}$, where the subscript indicates the direction of the $k$ vector and $z$ is the growth direction. An unstrained valence band offset of $0.6 \mathrm{eV}$ was used for this calculation. 


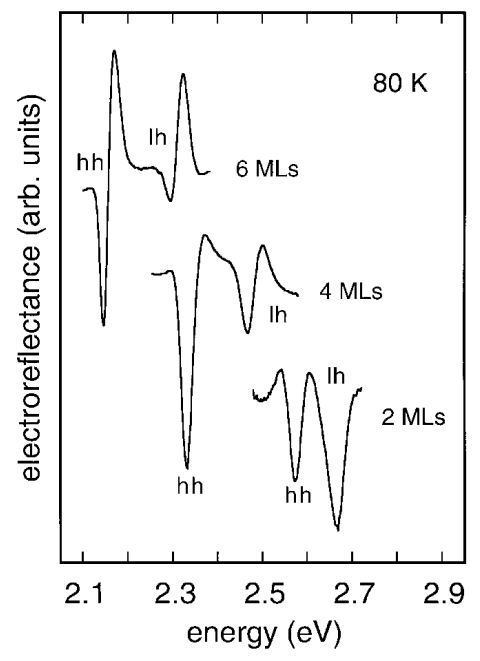

FIG. 2. Electroreflectance spectra for three GaAs quantum wells. The quantum well thickness is expressed in monolayers (MLs). Transitions between the $\Gamma$ band and heavy and light holes (labeled by hh and lh, respectively) are observed.

tinuity in the $X$ band is almost zero, which is similar to $\mathrm{Ge}$ strained to $\mathrm{Si}^{5}$ For electrons belonging to the $\Gamma$ band the GaAs forms a well about $0.5 \mathrm{eV}$ deep.

Figure 2 shows the ER spectra of three samples containing GaAs QWs having thicknesses of 2, 4, and $6 \mathrm{ML}$, respectively, taken at a temperature of $80 \mathrm{~K}$. Clear spectral features are seen, which are attributed to transitions between electrons in the $\Gamma$ conduction band and holes in the valence band. The higher energy transitions $(\Gamma-\mathrm{lh})$ are related to light holes and the lower energy transitions $(\Gamma-\mathrm{hh})$ are related to heavy holes. An energy shift with decreasing layer thickness is clearly seen, which we attribute to a quantum size effect. Moreover, the separation between heavy hole and light hole energies increases with increasing thickness of the GaAs QW. This is natural for thin quantum wells since in the limit of zero quantum well thickness (as well as for infinite quantum well thickness) the heavy hole and the light hole have the same energy. No ER was observed from transitions involving the $X$ band minima, presumably due to a low absorption cross-section of these indirect minima.

Figure 3 shows the PL spectra for the complete set of samples having well thicknesses between 1 and $6 \mathrm{ML}$. The measurement temperature was $7 \mathrm{~K}$ and an excitation wavelength of $363 \mathrm{~nm}$ was used. For this wavelength the penetration depth is of the order of the epitaxial layer thickness. An excitation power density of about $100 \mathrm{~W} / \mathrm{cm}^{2}$ was used. The emission is related to electrons in an $X_{x y}$ minimum recombining with heavy holes (labeled $X-\mathrm{hh}$ ). Here we also observe an energy shift, with higher transition energies for narrower QWs. For thicknesses below $6 \mathrm{ML}$ the emission shows three peaks. This is more clear for the sample with a $\mathrm{QW}$ thickness of $5 \mathrm{ML}$. The energy differences between the corresponding transitions are 12 and $32 \mathrm{meV}$, respectively, and they are nearly independent of layer thickness. Thus we cannot attribute these peaks as being due to monolayer fluctuations, but instead we attribute the lower energy peaks to phonon-assisted transitions, which is typical for indirect transitions. The energies of the phonons most closely correspond

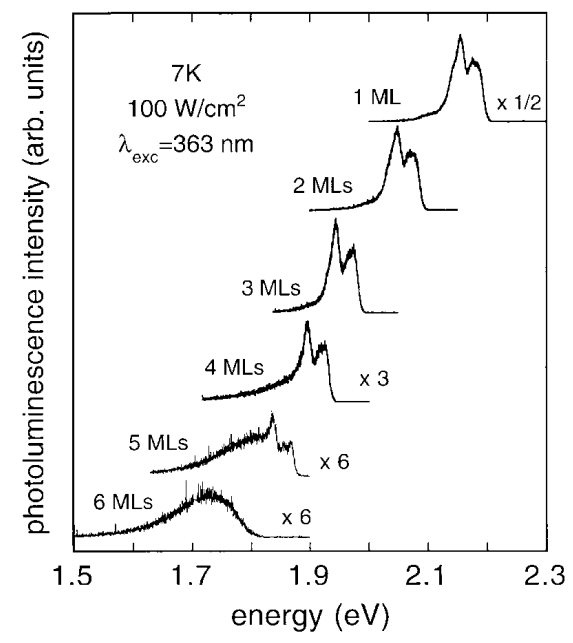

FIG. 3. Photoluminescence spectra of the emission from the quantum wells. Different magnifications of the spectra are used in the figure, since the emission intensity decreases for thicker quantum wells.

to $\mathrm{TA}^{x}$ and $\mathrm{LA}^{x}$ phonon energies in GaP. Phonon-assisted transitions involving $\mathrm{LA}^{x}$ and $\mathrm{TA}^{x}$ phonons (instead of the more commonly occurring LO phonons) have previously been observed in indirect GaAsP, both bulk ${ }^{17}$ as well as strained to $\mathrm{GaP}^{8}{ }^{8}$ The relative intensity of the no-phonon line decreases with decreasing thickness of the layers, becoming difficult to resolve for the thinnest QWs. This may be related to the increasing separation between $\Gamma$ states and $X$ states for narrower QWs. If the $\Gamma$ states are close to the $X$ states an in-mixing of the $\Gamma$ states into the $X$ states, due to e.g., interface roughness, will give a significant no-phonon transition. ${ }^{8}$ The total emission intensity increases almost linearly with increasing excitation power density and the emission intensity decreases with increasing QW thickness. We speculate that the influence of interface roughness is larger for smaller QWs which will increase the transition probabilities.

At the low energy side of the peak there is a broad tail. Photoluminescence spectra taken at higher temperatures (typically $30 \mathrm{~K}$ ) show only the broad emission. For the $5 \mathrm{ML}$ QW the relative intensities of the narrow peaks are small compared to that of the broad emission and for the $6 \mathrm{ML}$ QW no narrow peaks are observed. This broad emission is attributed to a defect in the strained layer. No emission from the bulk GaP is seen, indicating more efficient trapping of the carriers by the QWs than by defects in the GaP.

Figure 4 shows the observed transition energies, as well as the calculated transition energies, using three different values of the $\operatorname{VBO}(0.5,0.6$, and $0.7 \mathrm{eV})$. The experimental values are in all cases slightly smaller for the transition involving the $\Gamma$ band and the discrepancy increases with decreasing QW thickness. The agreement with theory is best for the highest VBO. However, the transitions involving the $X$ band introduce a constraint. For a VBO higher than about $0.6 \mathrm{eV}$, the GaAs layer turns type II, and the $X$-related electrons are no longer confined in the quantum well. In order to calculate the transitions involving the $X$ band we have calculated the confinement energy of the holes separately (using eight bands). For the values of the VBO in which the QW is type I the confinement of the $X$ electrons was calculated using a single-band model, using an effective mass of 0.25 


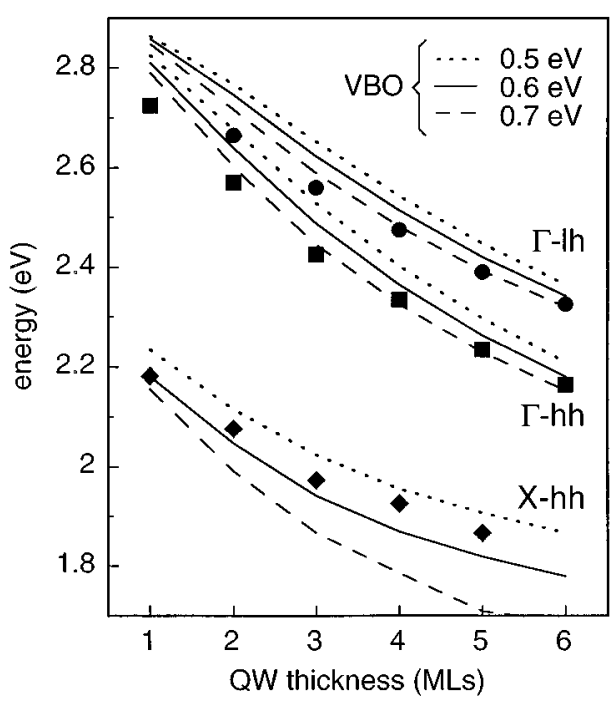

FIG. 4. A diagram of the observed transition energies as well as the calculated transition energies. Three different values for the unstrained valence band offset (VBO), indicated in the figure, have been used.

$m_{0}$ (where $m_{0}$ is the electron mass). The agreement with this experiment involving $X$ electrons is best using a VBO of 0.6 $\mathrm{eV}$. A VBO of $0.7 \mathrm{eV}$ (suggested by the experiment involving $\Gamma$ electron transitions) gives too low energies, since in this case a type II situation occurs and the transitions are between $X$ electrons in the GaP and holes in the GaAs. It has to be remembered that the deformation potential $E_{2}$, which gives the splitting of the $X$ conduction band under strain, is not very well known, and a different value can easily change the calculations for the $X$ transitions, when the QW is type I. However, changing $E_{2}$ cannot increase the transition energy for a type II situation, and we thus find that the upper limit of the VBO is about $0.6 \mathrm{eV}$, which also fits the experiments on the $\Gamma$ transitions reasonably well. The sensitivity to small (10\%) changes in other deformation potentials is quite low and do not change our conclusions. Another uncertainty in the determination of the $\mathrm{VBO}$ is the influence of intermixing at the interfaces. Based on estimations of the intermixing coming from Raman scattering data, ${ }^{12}$ we calculate this effect to give a blueshift of the transitions relating to the $\Gamma$ band, of about $0.05 \mathrm{eV}$. The actual thickness of the layers may also be somewhat different from the nominal thickness. Such an effect would affect the thinner QWs the most. We would like to remark that the observation of both $X$ - and
$\Gamma$-related transitions gives a more reliable value of the VBO than that based on only one of these sets of transitions. The obtained value of the VBO is in reasonable agreement with calculations of the $\mathrm{VBO}^{18}$ as well as with experiments based on emission from transition metal impurities in GaAsP, ${ }^{19}$ which give a $\mathrm{VBO}$ of $0.5 \mathrm{eV}$ and $0.4-0.5 \mathrm{eV}$, respectively.

In conclusion, we have studied both the $\Gamma$ band and the $X$ band related transitions of GaAs strained to GaP. The thicknesses of the GaAs QWs have been varied in steps of 1 ML between 1 and 6 ML. Using this rather complete experimental information in conjunction with theoretical modeling, we determine an unstrained valence band offset of about 0.6 $\mathrm{eV}$, giving a strained valence band offset of about $0.7 \mathrm{eV}$.

This work has been supported by the Spanish Comisión Interministerial de Ciencia y Tecnología under Project No. MAT95-0966-C02-01 and by the Human Capital and Mobility project HYPOCRATES. Professor L. Samuelson and Dr. M. S. Miller are thanked for helpful discussions, and Professor D. Gershoni is thanked for help with the computer program.

${ }^{1}$ G. C. Osbourn, J. Vac. Sci. Technol. B 1, 379 (1983).

${ }^{2}$ J. J. Rosenberg, M. Benlamri, P. D. Kirchner, J. M. Woodall, and G. D. Pettit, IEEE Electron Device Lett. EDL-6, 491 (1985).

${ }^{3}$ E. Yablonovitch and E. O. Kane, IEEE J. Lightwave Technol. LT-4, 961 (1986).

${ }^{4}$ P. G. Eliseev, B. N. Sverdlov, and N. Shokhudzhaev, Sov. J. Quantum Electron. 14, 1120 (1984).

${ }^{5}$ J. Olajos, J. Engvall, H. Grimmeiss, M. Gail, G. Abstreiter, H. Presting, and H. Kibbel, Phys. Rev. B 54, 1922 (1996).

${ }^{6}$ J. Arriaga, G. Armelles, M. C. Muñoz, J. M. Rodríguez, P. Castrillo, M. Recio, V. R. Velasco, F. Briones, and F. García-Moliner, Phys. Rev. B 43, 2050 (1991).

${ }^{7}$ M. Recio, G. Armelles, J. Meléndez, and F. Briones, J. Appl. Phys. 67, 2044 (1990).

${ }^{8}$ M.-E. Pistol, M. Leys, and L. Samuelson, Phys. Rev. B 37, 4664 (1988).

${ }^{9}$ D. Gershoni, C. H. Henry, and G. Baraff, IEEE J. Quantum Electron. 29, 2433 (1993)

${ }^{10}$ F. Briones, L. González, and A. Ruiz, Appl. Phys. A 49, 729 (1989).

${ }^{11}$ A. Mazuelas, L. González, F. A. Ponce, L. Tapfer, and F. Briones, J. Cryst. Growth 131, 465 (1993).

${ }^{12}$ P. Castrillo, G. Armelles, J. P. Silveira, and F. Briones (to be published).

${ }^{13}$ M.-E. Pistol, M. Gerling, D. Hessman, and L. Samuelson, Phys. Rev. B 45, 3628 (1992).

${ }^{14}$ Physics of Group-IV Elements and III-V Compounds, edited by O. Madelung, Landolt-Börnstein (Springer, Heidelberg, 1982), Vol. 17a.

${ }^{15}$ D. J. Wolford and J. A. Bradley, Solid State Commun. 53, 1069 (1985).

${ }^{16}$ C. N. Yeh, L. E. McNeil, R. E. Nahory, and R. Bhat, Phys. Rev. B 52, 14 682 (1995).

${ }^{17}$ Shui Lai and M. V. Klein, Phys. Rev. Lett. 44, 1087 (1980).

${ }^{18}$ S.-H. Ke, R. Wang, and M. Huang, Phys. Rev. B 49, 10495 (1994).

${ }^{19}$ M.-E. Pistol, S. Nilsson, and L. Samuelson, Phys. Rev. B 38, 8293 (1988). 